\title{
An Imaging Study of a Ramification Pattern of Right Posterior Portal Vein Branch
}

\author{
Paschalis Gavriilidis $^{1 *}$, Shi Yuan ${ }^{2}$, Li Weixia ${ }^{3}$, Chai Limin ${ }^{3}$ and Shen Baiyong ${ }^{2}$
}

${ }^{1}$ Department of Surgical Oncology, Theageneio Anticancer Hospital, Thessaloniki, Greece

${ }^{2}$ Department of Hepatobiliary and pancreatic surgery

${ }^{3}$ Department of Radiology, Ruijin Hospital, Shanghai Jiaotong University School of Medicine, Shanghai 200025, China

\section{Abstract}

Background: Our aim was to study the ramification pattern of the Right posterior portal vein branch and to compare our findings to Couinaud classification of the liver.

Study design: The study protocol was approved by the institutional review board and written informed consent was obtained from all patients before procedures were performed Our study includes 104 patients, all were Chinese who underwent helical Computed Tomography during Arterial Portography (CTAP). Subsequently, 3 dimensional portograms were reconstructed to verify the ramification patterns of the portal vein. Portal branching patterns of the right hemiliver were assessed.

Results: In all 104 patients examined, three distinct types of anatomical branching patterns of the Right posterior portal vein branch were observed:

- Type I: In 52 patients out of 104 (50\%), the Right posterior portal branch represents a single trunk forming an arch like pattern and sending multiple branches to segment 6 and segment 7, see Figure 1 and Figure 2 .

- Type II: In 9 out of 104 patients (9\%), the Right posterior portal vein branch showed trifurcation into posterosuperior, posteroinferior, and intermediate branches. The intermediate branch occupied the segmental border between segments S6 and S7, see Figure 3 and Figure 4.

- Type III: In 43 out of 104 patients (41\%), the Right posterior portal branch bifurcates into Posterosuperior P7 and Posteroinferior P6 third order portal branches as described by C. Couinaud in his homonymous segmental liver classification, see Figure 5 and Figure 6.

Conclusions: According to Couinaud classification, the Right posterior portal branch bifurcates into third order Postero superior P7 and Posteroiferior P6 portal branches to supply segments S7 and S6.

Our findings showed however, that it happened only in $41 \%$ of the cases. In $50 \%$ of the findings, the right posterior portal branch represented a single trunk forming an arch like pattern sending multiple branches. The smallest group of $9 \%$ showed trifurcation into posterosuperior, posteroinferior, and intermediate branches.

Therefore we conclude that there is a discrepancy between the Atlas model of liver segmentation and our imaging findings. Based on these findings we can consider a right lateral sector as a unique segment in $50 \%$ of cases.

Keywords: Right posterior portal vein branch; Right lateral sector; Liver segmental anatomy; Right hemiliver

\section{Introduction}

Our aim was to study the ramification pattern of the Right posterior portal vein branch and to compare our findings to Couinaud classification of the liver.

Though several scientists (Hjortso 1951; Elias \& Petty 1952; Healey \& Schroy 1953; Goldsmith \& Woodburne 1957) $[18,19,20,10]$ have conducted research on the anatomical division of liver, the segmental division of the liver based on portal vein ramification pattern proposed by Couinaud (1957) is the universally accepted.

In our days, the majority of the surgeons conceptualize the segmental anatomy of the liver in the manner described by Couinaud [1]. The three main hepatic veins divide the liver into four sectors which are designated as the left lateral, left paramedian, right paramedian and right lateral sectors based on portal ramifications.

These 4 sectors are each supplied by second order portal branches: the left lateral sector is supplied by the left laterosuperior portal vein, the left paramedian sector by the umbilical portion of the left portal vein, the right paramedian sector by the right anterior portal vein and the right lateral sector by the right posterior portal vein. Each of the eight segments of the liver is defined by its distinct and separate arterial and portal supply as well as its biliary drainage (portal pedicle). The portal pedicles are surrounded by Glisson's sheath.

In our multimodality meeting procedures such as US, CT, MR images and reconstructed 3D images of the portal vein tree with the help of the helical tomography, help us to delineate preoperatively the

*Corresponding author: Paschalis Gavrilidis MD, Department of Surgical Oncology, Theageneio Anticancer Hospital, Thessaloniki, Greece, Tel: +30 6977826253; Fax / Tel: +30 2310 898314; E-mail: pgav7461@yahoo.com

Received Janurary 05, 2011; Accepted February 02, 2011; Published February 04, 2011

Citation: Gavriilidis P, Yuan S, Weixia L, Limin C, Baiyong S (2011) An Imaging Study of a Ramification Pattern of Right Posterior Portal Vein Branch. J Cytol Histo 1:110. doi:10.4172/2157-7099.1000110

Copyright: (C) 2011 Gavriilidis P, et al. This is an open-access article distributed under the terms of the Creative Commons Attribution License, which permits unrestricted use, distribution, and reproduction in any medium, provided the original author and source are credited. 
sectoral and the segmental anatomy of the liver and accordingly our excisions. During the past decade there was an abundant research for delineating sectoral and segmental anatomy on US, CT, MR images [2$8,15]$. Advances in helical tomography have allowed the reconstruction of accurate and realistic 3D images from arbitrary angles [9]. During our interventions in the right hemiliver we encountered ramification patterns of the right posterior portal branch that were in discrepancy with the Atlas model. These operative findings triggered our interest to study preoperatively with 3D reconstructed images the ramification patterns of the Right posterior portal branch.

\section{Methods}

\section{Patients}

Between Mar 2006 and Nov 2006138 consecutive patients all were Chinese underwent CT scans. Of these, 104 patients enrolled in our study group, had no previous surgery and ranged in age from 19 to 74 years (mean 51). The patients had the following diagnoses: pancreatic tumors $(n=41)$, liver tumors $(n=32)$, haemangiomas $(n=3)$, lithiasis of the choledochus $(n=6)$, liver cirrhosis $(n=14)$, cancer of the gallbladder $(n=4)$, hepatic cyst $(n=1)$, Donator $(n=1)$, tumor of the duodenum $(\mathrm{n}=2)$.

The study protocol was approved by the institutional review board and written informed consent was obtained from all patients before procedures were performed.

\section{CT Imaging}

All studies were performed using commercially available helical CT scanners (Somatom plus 4, Siemens, Erlangen, Germany; or Aquilion, Toshiba Medical Systems. For CTAP, $90 \mathrm{ml}$ iohexol (300mg of iodine per $\mathrm{ml}$ ) diluted to one half concentration with saline was injected at a rate of $3 \mathrm{ml} / \mathrm{s}$ during a CT scan of the entire liver. The scan delay after the start of iohexol injection was 20 seconds.

Neither serious nor minor complications occurred during and after procedures in any patient.

\section{Image processing and analysis}

Three dimensional (3D) images were reconstructed from CT data downloaded to an independent work station using software for perspective volume rendering (3D Virtuoso, Siemens). The original consecutive axial CT images and 3D images were blinded and independently interpreted by authors. Any discrepancies that occurred were resolved by consensus.

\section{Results}

\section{Right posterior portal branching patterns}

In all 104 patients examined, three distinct types of anatomical branching patterns of the Right posterior portal vein branch were observed:

- Type I: In 52 patients out of 104 (50\%), the Right posterior portal branch represents a single trunk forming an arch like pattern and sending multiple branches to segment 6 and segment 7, see Figure 1 and Figure 2.

- Type II: In 9 out of 104 patients (9\%), the Right posterior portal vein branch showed trifurcation into posterosuperior, posteroinferior, and intermediate branches. The intermediate branch occupied the segmental border between segments S6 and S7, see Figure 3 and Figure 4.

- Type III: In 43 out of 104 patients (41\%), the Right posterior portal

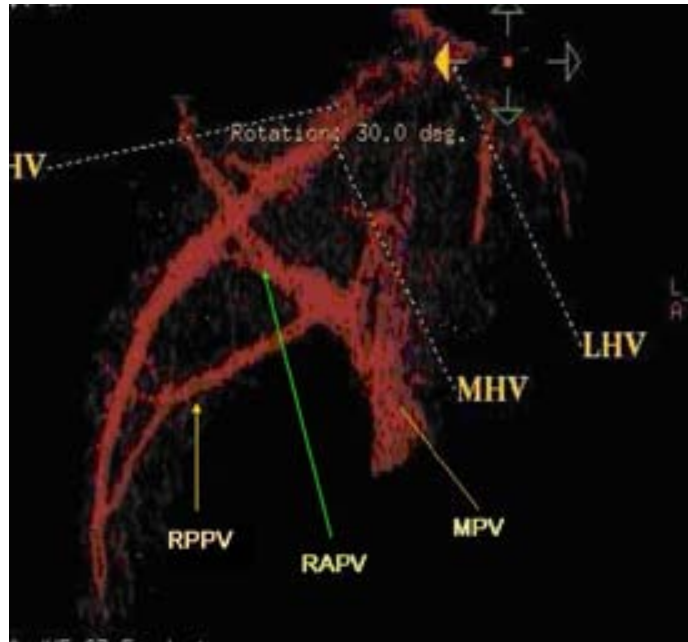

Figure 1: Type I unique right posterior portal branch Legend:

RAPV: Right anterior portal vein branch,

RPPV: Right posterior portal vein branch,

RHV: $\quad$ Right hepatic vein,

MHV: Middle hepatic vein,

LHV: $\quad$ Left hepatic vein,

MPV: $\quad$ Main portal vein

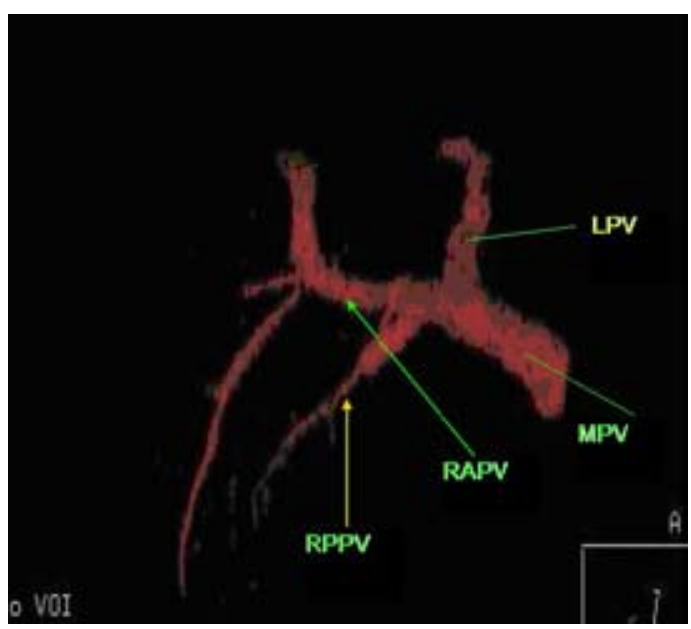

Figure 2: Type I unique right posterior portal branch Legend:

RAPV: $\quad$ Right anterior portal vein branch,

RPPV: Right posterior portal vein branch,

MPV: $\quad$ Main portal vein,

LPV: $\quad$ Left portal vein

branch bifurcates into Posterosuperior P7 and Posteroinferior P6 third order portal branches as described by C. Couinaud in his homonymous segmental liver classification, see Figure 5 and Figure 6.

\section{Discussion}

The classification proposed by Healey and Schroy [10] divided the liver into 4 segments which are designated as the lateral, medial, anterior, and posterior segments longitudinally along the three vertical fissures.

According to C. Couinaud, the liver is divided into 4 sectors which are designated as the left lateral, left paramedian, right paramedian and right lateral sectors based on portal ramifications. These 4 sectors 
Citation: Gavriilidis P, Yuan S, Weixia L, Limin C, Baiyong S (2011) An Imaging Study of a Ramification Pattern of Right Posterior Portal Vein Branch. J Cytol Histol 1:110. doi:10.4172/2157-7099.1000110

are each supplied by second order portal branches. That is, the left lateral sector is supplied by the left laterosuperior portal vein, the left paramedian sector by the umbilical portion, the right paramedian sector by the right anterior portal vein, and the right lateral sector by the right posterior portal vein.

Subsequently, the liver is divided in 8 segments by adding the caudate lobe as segment 1 . Each of the eight liver segments is defined by its distinct and separate arterial and portal supply as well as its biliary drainage (portal pedicle).

The portal pedicles are surrounded by the vasculobiliary sheath, distinguishing this structure from the hepatic veins. An important difference needs to be noted is that no intrahepatic anastomoses exist between the portal, arterial, and biliary structures of adjacent segments whereas the hepatic veins have large and numerous intrahepatic anastomoses. In addition, several segments are bordered by the same

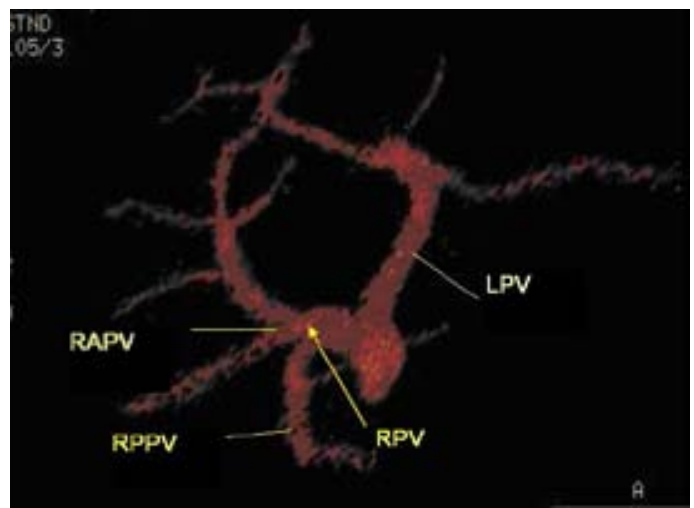

Figure 3: Type II Trifurcation

Legend:

RAPV: Right anterior portal vein branch

RPPV: $\quad$ Right posterior portal vein branch,

RPV: $\quad$ Right portal vein,

LPV: $\quad$ Left portal vein,

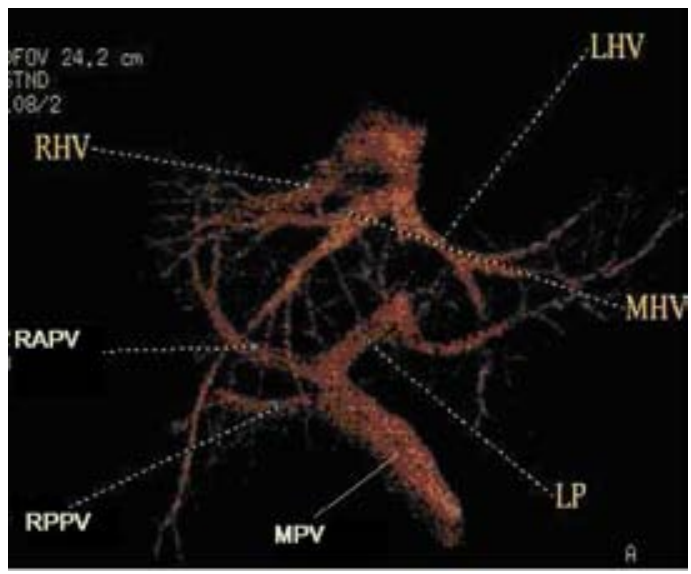

Figure 4: Type II Trifurcation

\section{Legend:}

RAPV: Right anterior portal vein branch,

RPPV: Right posterior portal vein branch,

RHV: $\quad$ Right hepatic vein,

MHV: $\quad$ Middle hepatic vein

LHV: $\quad$ Left hepatic vein,

LP: Left portal branch,

MPV: $\quad$ Main portal vein

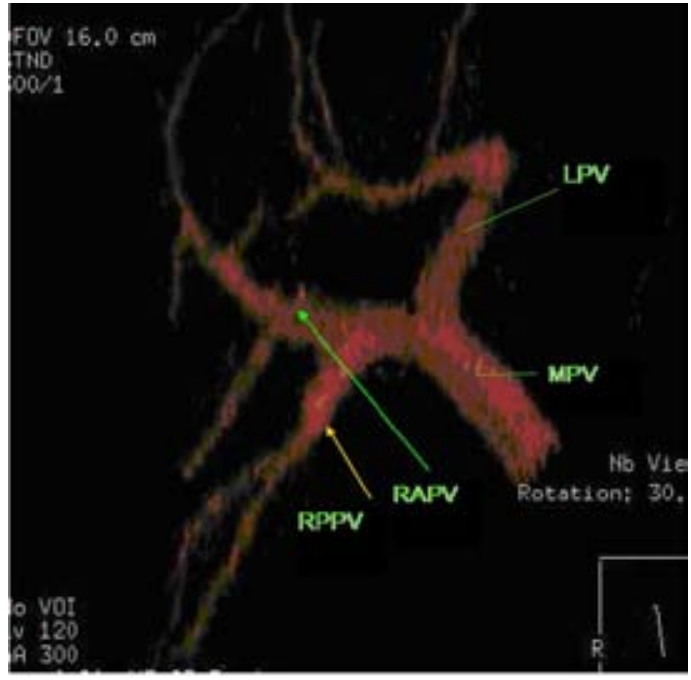

Figure 5: Type III or Couinaud classification Legend:

RAPV: Right anterior portal vein branch,

RPPV: Right posterior portal vein branch,

LPV: $\quad$ Left portal vein,

MPV: $\quad$ Main portal vein

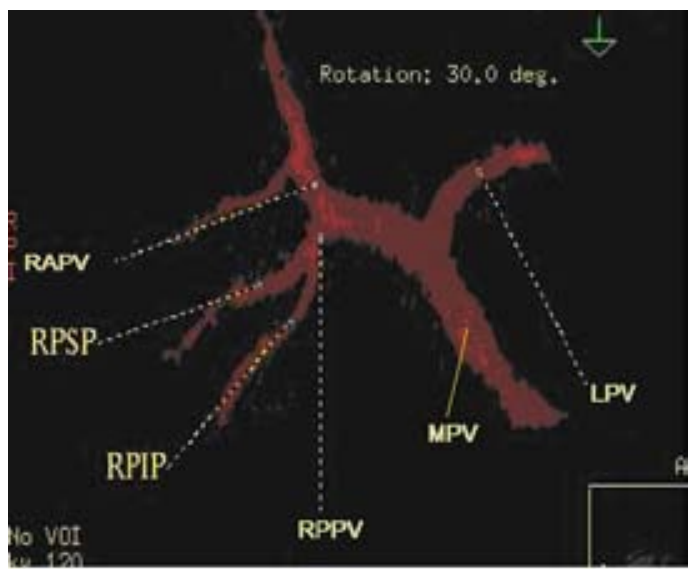

Figure 6: Type III or Couinaud classification Legend:

RAPV: Right anterior portal vein branch,

RPPV: $\quad$ Right posterior portal vein branch,

RPSP: Right posterosuperior portal branch,

RPIP: Right posteroinferior portal branch,

LPV: $\quad$ Left portal vein

MPV: $\quad$ Main portal vein

hepatic vein and the hepatic veins have abundant anatomic variations as shown in several studies $[12,13]$.

Based on the above we can understand how important is the detailed and granulated knowledge of the liver segmental anatomy for performing safe hepatectomies and segmentectomies. Hence any blind incision will inevitably lead to depriving the region of its arterial and portal blood supply and the creation of bile stasis or leakage as a result from a lack of intrahepatic anastomoses between the portal, arterial and biliary structures of adjacent segments.

Our finding showed that there is a "hole" in the Atlas model of the liver segmentation. Only $41 \%$ of the cases had the Couinaud similar pattern classification. In $9 \%$ of the cases the right posterior portal 
Citation: Gavrilidis P, Yuan S, Weixia L, Limin C, Baiyong S (2011) An Imaging Study of a Ramification Pattern of Right Posterior Portal Vein Branch. J Cytol Histol 1:110. doi:10.4172/2157-7099.1000110

branch trifurcates and in the remainder $50 \%$ of the cases, the right posterior portal branch does not bifurcate into posterosuperior P7 and posteroinferior P6 branch but represents a single truck forming an arch like pattern sending multiple branches. This characteristic defines the right lateral sector as unique segment and changes the whole concept of the Couinaud classification. Based on these characteristics, Akihiro Cho et al., proposed a reclassification of the liver anatomy [15].

Due to this discrepancy, we need to individualize the segmental liver anatomy of every patient by using helical tomograms and 3D reconstructed portograms and by knowing the real borders of the segments we will be able to perform safer segmental hepatectomies and harvest safely sector for living related liver transplantation [16]. Comparing our findings to the Japanese Cho [15] and to the Indian Arora et al. [17], we can conclude that this anatomic variation is a common finding in the Asian population. New studies comparing Asian, Caucasian and African racial groups need to be done.

Surgeons must not march as a bunch of lemmings into a sea of intellectual acceptance.

\section{References}

1. Couinaud C. Surgical Anatomy of the Liver Revisited. Paris, 1989

2. Mukai JK, Stack CM, Turner DA, Gould RJ, Petasnick JP, et al. (1987) Imaging of surgically relevant hepatic vascular and segmental anatomy. Normal anatomy. AJR Am J Roentgenol 149: 287-292.

3. Sugarbaker PH, Nelson RC, Murray DR, Chezmar JL, Bernardino ME (1990) A segmental approach to computerized tomographic portography for hepatic resection. Surg Gynecol Obstet 171: 189-195.

4. Saxton CC, Zeman RK (1983) Correlation of computed tomography, sonography, and gross anatomy of the liver. AJR Am J Roentgenol 141: 711718.

5. Nelson RC, Chezmar JL, Sugarbaker PH, Murray DR, Bernadini ME (1990) Preoperative localization of focal liver lesions to specific liver segments: utility of CT during arterial portography. Radiology 176: 89-94.

6. Lafortune M, Madore E, Patriquin H, Breton G (1991) Segmental anatomy of the liver: a sonographic approach to the Couinaud nomenclature. Radiology 181: 443-448.
7. Soyer P, Roche A, Gad M, Shapeero L, Breittmayer F, et al. (1991) Preoperative segmental localization of hepatic metastases: utility of three-dimensional CT during arterial portography. Radiology180: 653-658.

8. Gazelle GS, Haaga JR (1992) Hepatic neoplasms: surgically relevant segmenta anatomy and imaging techniques. AJR Am J Roentgenol 158: 1015-1018.

9. Rubin GD, Dake MD, Napel SA, McDonnell CH, Jeffrey RB Jr (1993) Threedimensional spiral CT angiography of the abdomen: initial clinical experience. Radiology 186: 147-152.

10. Healey JE Jr, Schroy PC (1953) An Anatomy of the biliary ducts within the human liver; analysis of the prevailing pattern of branchings and the major variations of the biliary ducts. AMA Arch Surg 66: 599-616.

11. Castaing D, Adam R, Azoulay D (2006) Chirurgie du foie et de l'hypertension portale. Masson Paris.

12. Makuuchi M, Hasegawa H, Yamazaki S, Bandai Y, Watanabe G, et al. (1983) The inferior right hepatic vein: ultrasonic demonstration. Radiology 148: 213 217.

13. Hardy KJ (1972) The hepatic veins. Aust N Z J Surg 42: 11-14.

14. Erbay N, Raptopoulos V, Pomfert EA, Kamel IR, Kruskal JB (2003) Living dono liver transplantation in adults : vascular variants important in surgical planning for donors and recipients. AJR Am J Roentgenol $181: 109-114$.

15. Cho A, Okazumi S, Miyazawa Y, Makino H, Miura F, et al. (2005) Proposal for a reclassification of liver based anatomy on portal ramifications. The American Journal of Surgery 189:195-199.

16. Kim SH, Suh KS, Kim SB, Lee HJ, Lee KU (2003) Adult living donor liver transplantation using right posterior segment. Tranapl Int 16: 689-691.

17. Arora J, Kapur V, Kakkar A, Dixit PC (2003) Ramification pattern of portal vein in right lobe of liver- A corrosion cast study. J Anat Soc India 52: 12-14.

18. Hjortsjo CH (1951) The topography of the intra-hepatic duct system. Acta Anat (Basel) 11: 599-615

19. Elias H, Petty D (1952) Gross anatomy of the blood vessels and ducts within the human liver. Am J Anat 90: 59-111.

20. Goldsmith NA, Woodburne RT (1957) The Surgical anatomy pertaining to liver resection. Surg Gynecol Obstet 105: 310-318. 